\title{
Auto Perceção do Desempenho da Medicina Centrada na Pessoa em Medicina Geral e Familiar: Criação de Um Instrumento de Medição
}

\section{Self-Awareness of Performing Patient-Centered Medicine in General Practice / Family Medicine: Development of a Measurement Scale}

\author{
Luiz Miguel SANTIAGO $\triangle^{1,2}$, José Augusto SIMÕES², Martinha VALE¹, Elleni de FARIA ${ }^{1}$, Pedro Lopes FERREIRA³, \\ Inês ROSENDO ${ }^{1}$
}

Acta Med Port 2020 Jun;33(6):407-414 - https://doi.org/10.20344/amp.11742

\section{RESUMO}

Introdução: A Medicina Centrada na Pessoa é um método e modelo de prática permitindo ganhos para o médico e o paciente, devendo a sua prática ser avaliada para fins de desenvolvimento profissional contínuo e educação médica continuada. O objectivo deste estudo foi construir, determinar a fiabilidade e a validade de um instrumento capaz de aferir a auto perceção genérica da prática médica segundo a medicina centrada na pessoa no ambiente de medicina geral e familiar.

Material e Métodos: Uma primeira versão de um questionário segundo as quatro dimensões do método clínico centrado na pessoa foi revista por um grupo focal garantindo a validade de conteúdo. O questionário final engloba 22 itens, utilizando para resposta uma escala de Likert com quatro opções. A análise fatorial permitiu confirmar as dimensões definidas por Moira Stewart, tendo também sido determinada a consistência interna, a reprodutibilidade por teste-reteste e a correlação item-total. A aplicação online a uma amostra de 905 médicos de medicina geral e familiar permitiu testar as validades de constructo e de critério.

Resultados: O instrumento de medição inclui quatro dimensões: (i) explorar a saúde, a doença e a experiência de doença, (ii) investir na relação médico-doente; (iii) procurar entendimento; e (iv) compreender a pessoa como um todo. A consistência interna foi demonstrada com um alfa de Cronbach global de 0,892 , variando entre 0,783 a 0,844 para todas as dimensões. A reprodutibilidade teste-reteste obteve um valor de correlação intraclasse entre 0,678 e 1,000. As correlações item-total variaram entre 0,457 e 0,870 . As mulheres médicas estão mais sensibilizadas do que os seus colegas no que respeita à procura de entendimento com o doente e os médicos mais novos são os mais sensíveis à abordagem da doença através da anamnese e à procura de entendimento com o doente. Os especialistas demonstraram ter mais cuidado com a anamnese e com o facto de encarar o doente como um todo, e os profissionais que trabalham numa unidade de Saúde Familiar são os que procuram um melhor entendimento com a pessoa. Por fim, a formação específica sobre medicina centrada na pessoa e sobre consulta em medicina centrada na pessoa demonstraram ter um impacto positivo em todas as dimensões da medicina centrada na pessoa e isso é reconhecido pelos próprios profissionais.

Discussão: A avaliação da auto perceção de desempenhar medicina centrada na pessoa é agora possível.

Conclusão: O presente questionário apresenta boa fiabilidade e validade permitindo ao médico verificar quais as principais insuficiências bem como desenvolver formação específica.

Palavras-chave: Cuidados de saúde primários; Fiabilidade; Medicina Centrada na Pessoa; Medicina Geral e Familiar; Método Clínico Centrado na Pessoa; Questionário; Relação Médico-Doente

\section{ABSTRACT}

Introduction: Patient Centred Medicine is a method and a model of practicing allowing gains for both the doctor and the patient. Its practice must be evaluated for purposes of continuous professional development and continuous medical education. The aim of this study was to create an instrument focused in measuring the practice based on person centered medicine in general and family medicine, as well as in determining its reliability and validity.

Material and Methods: A first version of a questionnaire according to the four dimensions of the patient- centred clinical method was revised in a focus group providing the content validity. The final questionnaire includes 22 items, using a Likert scale with four response options. Factorial analysis made it possible to confirm the dimensions defined by Moira Stewart, and internal consistency, test-retest reproducibility and item-total correlations were determined. The online implementation of the questionnaire to a sample of 905 family doctors guaranteed the construct and criterion validities.

Results: The measurement instrument includes four dimensions: (i) exploring health, disease and the illness experience; (ii) investing in the doctor-patient relationship; (iii) seeking understanding; and (iv) understanding the whole person. The internal consistency was demonstrated with a global Cronbach's alpha of 0.892 , varying between 0.783 and 0.844 for all dimensions. The test-retest reproducibility obtained an intraclass correlation value between 0.678 and 1.000 . The item-total correlations varied between 0.457 and 0.870 . Women doctors are more aware than their colleagues about seeking understanding with the person, and young doctors are more susceptible to approach the disease through history and to seek understanding with the patient. Specialist physicians have shown to be more careful with the history and with viewing the patient as a whole and the professionals who work in a Family Health Unit are those who seek a better understanding with the person. Finally, specific training about person centered medicine and about consultation in person centered medicine demonstrated a positive impact in all dimensions of the person-centred medicine and this is acknowledged

1. Clínica Universitária de Medicina Geral e Familiar. Faculdade de Medicina. Universidade de Coimbra. Coimbra. Portugal.

2. Centro de Estudos e Investigação em Saúde. Faculdade de Medicina. Universidade de Coimbra. Coimbra. Portugal.

3. Centro de Estudos e Investigação em Saúde. Universidade de Coimbra. Coimbra. Portugal.

$\square$ Autor correspondente: Luiz Miguel Santiago. Imsantiago@netcabo.pt

Recebido: 27 de dezembro de 2018 - Aceite: 31 de outubro de 2019 | Copyright @ Ordem dos Médicos 2020 
by professionals.

Discussion: The assessment of self-perception of person-centred medicine is now possible.

Conclusion: The questionnaire presents good reliability and validity, thus allowing doctors to assess their main weaknesses, as well as enabling the development of specific training.

Keywords: Clinical Method Focused on the Person; Doctor-Patient Relationship; General and Family Medicine; Person-Centered Medicine; Primary health care; Questionnaire; Reliability

\section{INTRODUÇÃO}

A medicina centrada na pessoa (MCP) $)^{1,2}$ é atualmente reconhecida como um método importante no exercício da medicina. ${ }^{3}$ De facto, existe uma relação entre a abordagem da MCP e uma maior satisfação das pessoas doentes e dos médicos que a sentem e a realizam. É também evidente uma maior redução de preocupações e da ansiedade, uma maior adesão à terapêutica proposta, uma melhoria da saúde mental e do estado funcional, uma diminuição das reclamações por negligência médica, uma redução dos custos e da utilização de consultas nos cuidados de saúde primários (CSP), o número de recorrências ao serviço de urgência e de hospitalizações, a requisição de exames complementares de diagnóstico, a referenciação para consultas de outras especialidades médicas e a melhoria da eficiência nos cuidados de saúde. ${ }^{4-9}$

Este conceito de MCP, introduzido por Balint em 1970, baseia-se no reconhecimento da pessoa que recorre aos cuidados de saúde na sua globalidade, considerando as suas experiências, valores, necessidades e preferências..$^{1,9}$ Defende a partilha do poder entre o médico e a pessoa, de forma a alcançar uma aliança terapêutica em que esta assume um papel ativo nas decisões terapêuticas e nas inerentes responsabilidades. Revoga o modelo biomédico paternalista, cujo foco é a doença enquanto entidade objetiva em que o médico ocupa uma posição de distanciamento e supremacia na tomada das decisões. ${ }^{3,10}$

Segundo Moira Stewart et al, ${ }^{3}$ esta abordagem centrada na pessoa é apresentada como um modelo e um método também denominado Método Clínico Centrado na Pessoa (MCCP). Fundamenta-se este método em quatro componentes dependentes e interrelacionadas: (1) explorar a saúde, a doença e a experiência da doença; (2) compreender a pessoa como um todo; (3) procurar entendimento; e (4) melhorar a relação médico-doente.

Pela abordagem da doença, através da anamnese, do exame físico e da perspetiva da pessoa sobre a sua saúde e a sua doença, bem como sobre a enfermidade ou dolência, são identificadas as suas ideias, os seus receios, as suas expectativas e o impacto funcional da sua situação clínica. Seguidamente, compreende-se a pessoa como um todo, tendo em conta a integração destes conceitos com a personalidade e os contextos familiar, laboral, cultural e social em que se encontra. Após esta fase, procura-se um entendimento com a pessoa, identificando-se os problemas, estabelecendo-se um plano terapêutico conjunto e determinando-se os papéis do médico e da pessoa. Por fim, investe-se na relação médico-doente através da construção de uma relação de confiança capacitante, empática, de compaixão e de uma partilha de poder que exige uma capacidade de entender aspetos do relacionamento, como a transferência e contratransferência, e ter consciência de si mesmo, sabendo-se que é um processo longo e que se vai construindo.

Têm sido realizados estudos com o propósito de determinar se a prática clínica atual está a ser orientada segundo este método e de identificar a perspetiva da pessoa que consulta o médico, ${ }^{11,12}$ assumindo o seu grau de satisfação como indicador de qualidade dos serviços de saúde. Importa agora saber como é que os médicos auto percecionam o seu próprio desempenho neste âmbito, com o intuito de iniciar posteriores ações de desenvolvimento profissional contínuo e de educação médica continuada em MCP e no MCCP e, além disto, para garantir que as atitudes do médico vão ao encontro das legitimas expetativas dos doentes.

Concomitantemente, os autores do MCCP e da MCP desenvolveram modelos teóricos de avaliação relativamente ao que acontece numa consulta. ${ }^{12}$ Porém, não há um instrumento genérico que permita verificar se, no contexto dos CSP ou em medicina geral e familiar (MGF) em Portugal, a prática se rege segundo estes enquadramentos.

Assim, o objetivo deste trabalho foi criar um instrumento capaz de medir a auto perceção genérica da prática médica de acordo com o MCCP, destinado aos médicos especialistas em MGF. Pretendeu-se também verificar a fiabilidade e a validade deste novo instrumento de medição.

\section{MATERIAL E MÉTODOS}

Para a realização desta investigação, foram essencialmente percorridas três fases: (1) elaboração do questionário de medição de adesão à MCCP; (2) teste da fiabilidade; e (3) teste de validade.

Este estudo obteve parecer positivo da Comissão de Ética da Administração Regional de Saúde do Centro e a aprovação da autora do MCCP, Moira Stewart. ${ }^{3}$

\section{Elaboração do questionário}

Com base nas quatro componentes do MCCP atrás referidas, nesta fase procedeu-se à criação de uma primeira lista de afirmações, diretamente resultantes das dimensões propostas por Moira Stewart e baseada em pesquisa bibliográfica e documental. Deste modo, para iniciar, realizou-se em setembro de 2016 um grupo focal com médicos convidados de ambos os sexos e de diversas idades e tempos de exercício da MGF, formadores e não formadores da especialidade. Após consentimento de todos os presentes, procedeu-se a um debate vídeo gravado sobre o material que havia sido previamente enviado contendo esta primeira versão da lista de afirmações e foi discutido qual o formato mais apropriado para lista de opções de resposta.

Concluído este grupo focal e consensualizadas todas 
as sugestões e propostas de alteração, obteve-se uma versão final que, posteriormente, foi sujeita a uma avaliação linguística por uma especialista.

\section{Fiabilidade}

Para abordar a fiabilidade, optou-se pelo teste da reprodutibilidade (estabilidade temporal), pela análise da consistência interna e pela determinação das correlações item-total. No que respeita a reprodutibilidade, realizou-se um teste-reteste, aplicando o questionário em dois tempos diferentes, com três dias de intervalo, a uma amostra de conveniência formada por médicos especialistas em MGF, de ambos os sexos, de diferentes grupos etários e tipos de unidades de saúde, a exercer as suas atividades profissionais nas Regiões de Saúde do Norte e do Centro. Esta consulta foi realizada, de forma anónima e confidencial, entre os meses de outubro e novembro de 2016. De modo a emparelhar os questionários preenchidos pelo mesmo médico, estes foram previamente numerados, tendo sido dado conhecimento aos médicos.

Para testar a consistência interna e a homogeneidade entre itens individuais e para determinar os valores das correlações item-total, o questionário obtido na etapa anterior foi aplicado online a uma amostra também utilizada posteriormente para a análise da validação deste instrumento de medição.

\section{Validade}

A análise da validade seguiu as três formas de validade reconhecidas cientificamente: (1) validade de conteúdo; (2) validade de construção; e (3) validade de critério. ${ }^{13}$

A validade de conteúdo pretende determinar o grau segundo o qual um instrumento de medição inclui os aspetos mais relevantes a serem medidos. No nosso caso contou, como mencionado anteriormente, com a colaboração de juízos de peritos sobre o conteúdo da medida. De facto, médicos de família distintos dos que contribuíram para a criação do instrumento de medição, avaliaram a clareza, a compreensão e o grau de redundância dos itens e das escalas. Além disto, foi também avaliado o conhecimento da inteligibilidade através de uma questão direta aquando da recolha do questionário.

A validade de construção foi testada através das relações lógicas existentes entre os vários itens que constituem o instrumento de medição. Para determinar os fatores subjacentes ao questionário e para, de certo modo, confirmar as dimensões propostas por Moira Stewart, procedeu-se a uma análise fatorial exploratória, tendo previamente sido aplicado o teste de $\mathrm{KMO}$ e o de esfericidade de Bartlett. ${ }^{14}$ Esta análise foi baseada em estimativas de componentes principais e foi submetida a uma rotação varimax para melhor se identificarem os fatores subjacentes aos dados. Para a seleção do número de fatores foi seguido o critério de Kaizer do valor próprio superior a um. ${ }^{14}$

Além disto, uma vez confirmadas as dimensões propostas pela autora, foram determinadas as médias das respostas correspondentes aos itens de cada dimensão e formuladas as seguintes duas hipóteses:

- $\mathrm{H}_{1}$ : A adesão à MCP não está dependente das características sexo e idade dos médicos;

- $\mathrm{H}_{2}$ : A adesão à MCP não está dependente da especialização ou do local de trabalho dos médicos.

A base de dados utilizada foi obtida em novembro de 2017 de uma aplicação online a um universo de 6460 médicos por iniciativa da Secção Regional do Centro da Ordem dos Médicos e das coordenações de internato das Regiões Centro e Norte. Apenas foram consideradas válidas as respostas com os 22 itens do questionário respondidos, tendo o cálculo do tamanho da amostra, para uma margem de erro de $5 \%$ e um nível de confiança de $95 \%$, sido de 308 respondentes.

A validade de critério analisa até que ponto as medições obtidas pelo instrumento estão relacionadas com as obtidas por um método de referência. No nosso caso, não havendo outro instrumento de medição especialmente desenhado para aferir a auto perceção da prática médica segundo a MCP, optou-se por analisar a diferença entre as respostas obtidas pelos médicos relativamente à frequência de formação específica sobre MCP (sim/não), à frequência de formação específica sobre consulta em MCP (sim/não) e se considera estar, ele próprio, a aplicar a MCP (sim/não). Foram assim definidas as seguintes três hipóteses:

- $\mathrm{H}_{3}$ : Os médicos com frequência de formação específica sobre MCP não apresentam valores diferentes dos que não têm esse tipo de formação;

- $\mathrm{H}_{4}$ : Os médicos com frequência de formação específica sobre consulta em MCP não apresentam valores diferentes dos que não têm esse tipo de formação;

- $\mathrm{H}_{5}$ : Os médicos que consideram estar a aplicar a MCP não apresentam valores diferentes dos que não consideram estar a aplicar este método.

\section{Análise de dados}

Para a determinação da consistência interna o indicador estatístico adotado foi o alfa de Cronbach, sendo aceites valores entre 0,7 e $0,9 .{ }^{15}$ Já no teste da reprodutibilidade foi utilizado o coeficiente ICC de correlação intraclasse. Um valor inferior a 0,5 é considerado fraco, entre 0,5 e 0,75 moderado, entre 0,75 e 0,9 bom, e acima de 0,9 excelente. ${ }^{16}$

Para testar as duas primeiras hipóteses, a variável sexo foi classificada em feminino/masculino e a idade foi agrupada em três grupos etários [ $\leq 35$ anos, (35 - 55 anos)], $\geq 65$ anos). Os médicos foram também agrupados em especialistas ou internos e o local de trabalho incluiu as unidades UCSP e USF.

Os métodos estatísticos utilizados nas cinco hipóteses foram o $t$ de Student e a ANOVA dependendo de a variável em causa ter dois ou mais possíveis valores. Foi assumido um erro de tipo I associado a uma probabilidade de 0,05. O software estatístico utilizado foi o SPSS v.19. 


\section{RESULTADOS}

\section{Elaboração do questionário}

Como referido anteriormente, a análise do livro de Moira Stewart conduziu inicialmente à elaboração de um questionário com 16 itens associados a três opções de resposta ('não', 'em parte' e 'sim'), divididas conceptualmente por quatro capítulos, correspondentes às dimensões da MCP e propostas pela autora. A este questionário, foram adicionadas três outras perguntas de carácter epidemiológico, concretamente: sexo, grupo etário e tipo de unidade de saúde em que trabalha.

Após a discussão do grupo focal, incluíram-se seis novos itens, tendo a versão final do questionário ficado com 22 questões. As opções de resposta foram consensualmente alteradas para quatro âncoras ('quase sempre', 'muitas vezes', 'poucas vezes' e 'raramente') para uma meIhor discriminação dos resultados. A estas âncoras foram atribuídos, respetivamente, os valores 1 a 4 , no sentido de uma menor pontuação significar uma prática mais próxima do MCP.

A formulação dos itens foi sempre feita na positiva, como forma de perceção da realização do método, em fidelidade ao modelo descrito por Moira Stewart et al. ${ }^{12} \mathrm{~A}$ Fig. 1 apresenta a versão final do questionário.

\section{Nas consultas de Medicina Geral e Familiar agendadas pelo consulente, costumo:}

\section{Resposta}

1. Deixar falar inicialmente a pessoa, sem interrupção, sobre os sinais e sintomas que motivam a sua vinda à consulta

2. Pedir à pessoa que fale sobre os seus receios e ideias acerca do que tem

3. Perguntar sobre as expectativas acerca do que possa resultar da consulta

4. Perguntar acerca da influência dos problemas na sua vida diária quer física quer emocionalmente

5. Inquirir sobre a sua perspetiva de ter uma doença

6. Saber da auto perceção de estado geral de saúde

7. Analisar a sua comunicação verbal e não-verbal

8. Realizar exame físico e/ou analítico acerca das queixas

9. Manter atualizado o conhecimento sobre a pessoa (formação, atividades laborais e extralaborais), religiosidade, pontos de interesse, rendimentos e aspirações.

10. Manter atualizado o conhecimento sobre a sua família (relações familiares, condições de habitação e rendimentos)

11. Manter atualizado o conhecimento sobre a ligação da pessoa à sociedade (voluntariado, cultura e política)

12. Elaborar com a pessoa a lista de problemas na consulta

13. Definir em conjunto as prioridades a resolver

14. Explicar o processo de tratamento que será realizado em conjunto e colaboração

15. Certificar-me de que a pessoa percebeu e concorda com os objetivos a atingir

16. Inquirir se percebeu o que deve ser feito para evitar piorar

17. Verificar que a pessoa percebeu a importância de cumprir as indicações para que se obtenham resultados

18. Observar os princípios da empatia médica tendo compaixão com a pessoa.

19. Tentar que a consulta dure o tempo necessário.

20. Demonstrar confiança nos meus conhecimentos e atitudes

21. Dar espaço e responder às dúvidas da pessoa

22. Ter comportamento que permita perceber em simultâneo o corpo e a mente do meu consulente

\begin{tabular}{|c|c|c|c|}
\hline $\begin{array}{l}\text { Quase } \\
\text { sempre }\end{array}$ & $\begin{array}{l}\text { Muitas } \\
\text { vezes }\end{array}$ & $\begin{array}{c}\text { Poucas } \\
\text { vezes }\end{array}$ & $\begin{array}{l}\text { Rara- } \\
\text { mente }\end{array}$ \\
\hline$\square_{1}$ & $\square 2$ & $\square 3$ & $\square_{4}$ \\
\hline$\square_{1}$ & $\square_{2}$ & $\square 3$ & $\square_{4}$ \\
\hline$\square_{1}$ & $\square 2$ & $\square_{3}$ & $\square_{4}$ \\
\hline$\square_{1}$ & $\square_{2}$ & $\square_{3}$ & $\square_{4}$ \\
\hline$\square_{1}$ & $\square 2$ & $\square_{3}$ & $\square_{4}$ \\
\hline$\square_{1}$ & $\square 2$ & $\square_{3}$ & $\square_{4}$ \\
\hline$\square_{1}$ & $\square_{2}$ & $\square_{3}$ & $\square_{4}$ \\
\hline$\square_{1}$ & $\square_{2}$ & $\square_{3}$ & $\square_{4}$ \\
\hline$\square_{1}$ & $\square_{2}$ & $\square_{3}$ & $\square_{4}$ \\
\hline$\square_{1}$ & $\square_{2}$ & $\square_{3}$ & $\square_{4}$ \\
\hline$\square_{1}$ & $\square_{2}$ & $\square_{3}$ & $\square_{4}$ \\
\hline$\square_{1}$ & $\square_{2}$ & $\square_{3}$ & $\square_{4}$ \\
\hline$\square_{1}$ & $\square 2$ & $\square_{3}$ & $\square_{4}$ \\
\hline$\square_{1}$ & $\square_{2}$ & $\square_{3}$ & $\square_{4}$ \\
\hline$\square_{1}$ & $\square 2$ & $\square 3$ & $\square_{4}$ \\
\hline$\square_{1}$ & $\square_{2}$ & $\square_{3}$ & $\square_{4}$ \\
\hline$\square_{1}$ & $\square_{2}$ & $\square_{3}$ & $\square_{4}$ \\
\hline$\square_{1}$ & $\square_{2}$ & $\square_{3}$ & $\square_{4}$ \\
\hline$\square_{1}$ & $\square_{2}$ & $\square_{3}$ & $\square_{4}$ \\
\hline$\square_{1}$ & $\square_{2}$ & $\square_{3}$ & $\square_{4}$ \\
\hline$\square_{1}$ & $\square_{2}$ & $\square_{3}$ & $\square_{4}$ \\
\hline$\square_{1}$ & $\square_{2}$ & $\square_{3}$ & $\square_{4}$ \\
\hline
\end{tabular}

Figura 1 - Versão final do questionário 


\section{Fiabilidade}

A reprodutibilidade, através de um teste-reteste, foi testada numa amostra de 63 inquéritos distribuídos e recebidos, não tendo havido qualquer exclusão por todos os itens estarem devidamente preenchidos. Tratou-se de uma amostra com $26(41,7 \%)$ respondentes do sexo masculino, $16(25,4 \%)$ com menos de 35 anos e $32(50,8 \%)$ com uma idade compreendida entre 35 e 55 anos. Em relação ao tipo de unidade de saúde, $25(39,7 \%)$ trabalhavam em UCSP e $38(60,3 \%)$ em USF.

A Tabela 1 apresenta os valores de ICC (coluna 3) correspondente a cada afirmação. Os valores encontrados podem ser considerados todos muito bons.

Entretanto, para avaliar a consistência interna e determinar as correlações item-total, recorremos à aplicação via internet de um questionário dirigido a um universo de 6460 médicos a quem foram aplicadas as questões que descreviam a prática MCP. Do questionário enviado obtiveram-se 996 respostas (15,4\%). Destas, estavam completas com os 22 itens respondidos e foram tratadas $905(90,8 \%)$ respostas, embora nem todas as variáveis sociodemográficas e laborais fossem completamente preenchidas, havendo neste caso uma percentagem de dados omissos de $7,0 \%$ a $12,6 \%$ (exceção foi o grupo etário que registou uma percentagem de $31,3 \%$ de dados omissos).

Na Tabela 2 são referidas as características da amostra final, que é sobretudo feminina $(65,5 \%)$, formada por jovens $(61,1 \%$ com 35 ou menos anos) trabalhando em USF $(67,0 \%)$, sem frequência de formação específica sobre MCP $(65,1 \%)$ nem de formação específica sobre consulta em MCP (68,5\%), mas julgando maioritariamente praticá-la $(79,9 \%)$.

\section{Validade}

A validade de conteúdo foi entretanto garantida através da intervenção do grupo focal que discutiu as questões que Ihe foram apresentadas, relativamente à sua clareza, compreensão e redundância.

Para confirmar as dimensões propostas pela autora, com base nas respostas às 22 afirmações, foi aplicada uma análise fatorial exploratória à base de dados composta pelas 905 respostas de um inquérito aplicado online.

Tabela 1 - Fiabilidade teste-reteste

\begin{tabular}{|c|c|c|c|}
\hline Item & & ICC & Item-Total \\
\hline 1 & $\begin{array}{l}\text { Deixar falar inicialmente a pessoa, sem interrupção, sobre os sinais e sintomas que motivam } \\
\text { a sua vinda à consulta }\end{array}$ & 0,746 & $0,506[2]$ \\
\hline 2 & Pedir à pessoa que fale sobre os seus receios e ideias acerca do que tem & 0,950 & $0,655[1]$ \\
\hline 3 & Perguntar sobre as expectativas acerca do que possa resultar da consulta & 0,964 & $0,715[1]$ \\
\hline 4 & $\begin{array}{l}\text { Perguntar acerca da influência dos problemas na sua vida diária quer física quer } \\
\text { emocionalmente }\end{array}$ & 0,678 & $0,671[1]$ \\
\hline 5 & Inquirir sobre a sua perspetiva de ter uma doença & 0,723 & $0,726[1]$ \\
\hline 6 & Saber da auto perceção de estado geral de saúde & 0,889 & $0,679[1]$ \\
\hline 7 & Analisar a sua comunicação verbal e não-verbal & 0,813 & $0,536[2]$ \\
\hline 8 & Realizar exame físico e/ou analítico acerca das queixas & 1,000 & $0,457[2]$ \\
\hline 9 & $\begin{array}{l}\text { Manter atualizado o conhecimento sobre a pessoa (formação, atividades laborais e extrala- } \\
\text { borais), religiosidade, pontos de interesse, rendimentos e aspirações. }\end{array}$ & 0,896 & $0,838[4]$ \\
\hline 10 & $\begin{array}{l}\text { Manter atualizado o conhecimento sobre a sua família (relações familiares, condições de } \\
\text { habitação e rendimentos) }\end{array}$ & 0,905 & $0,870[4]$ \\
\hline 11 & $\begin{array}{l}\text { Manter atualizado o conhecimento sobre a ligação da pessoa à sociedade (voluntariado, } \\
\text { cultura e política) }\end{array}$ & 0,917 & $0,830[4]$ \\
\hline 12 & Elaborar com a pessoa a lista de problemas na consulta & 0,839 & $0,717[1]$ \\
\hline 13 & Definir em conjunto as prioridades a resolver & 0,920 & $0,674[1]$ \\
\hline 14 & Explicar o processo de tratamento que será realizado em conjunto e colaboração & 0,727 & $0,766[3]$ \\
\hline 15 & Certificar-me de que a pessoa percebeu e concorda com os objetivos a atingir & 0,921 & $0,870[3]$ \\
\hline 16 & Inquirir se percebeu o que deve ser feito para evitar piorar & 0,846 & $0,849[3]$ \\
\hline 17 & $\begin{array}{l}\text { Verificar que a pessoa percebeu a importância de cumprir as indicações para que se obtenham } \\
\text { resultados }\end{array}$ & 0,743 & $0,816[3]$ \\
\hline 18 & Observar os princípios da empatia médica tendo compaixão com a pessoa. & 0,966 & $0,630[2]$ \\
\hline 19 & Tentar que a consulta dure o tempo necessário. & 0,970 & $0,661[2]$ \\
\hline 20 & Demonstrar confiança nos meus conhecimentos e atitudes & 1,000 & $0,736[2]$ \\
\hline 21 & Dar espaço e responder às dúvidas da pessoa & 0,848 & 0,746 [2] \\
\hline 22 & Ter comportamento que permita perceber em simultâneo o corpo e a mente do meu consulente & 0,979 & $0,755[2]$ \\
\hline
\end{tabular}


Tabela 2 - Características da amostra

\begin{tabular}{|c|c|c|c|}
\hline Variável & Valor & $\mathbf{n}$ & $\%$ \\
\hline \multirow[t]{2}{*}{ Sexo } & Masculino & 287 & 34,5 \\
\hline & Feminino & 545 & 65,5 \\
\hline \multirow[t]{3}{*}{ Idade } & $\leq 35$ anos & 380 & 61,1 \\
\hline & 36 a 55 anos & 162 & 26,0 \\
\hline & $\geq 56$ anos & 80 & 12,9 \\
\hline \multirow[t]{2}{*}{ Funções } & Especialista & 622 & 73,9 \\
\hline & Interno & 220 & 26,1 \\
\hline \multirow[t]{2}{*}{ Local de Trabalho } & UCSP & 261 & 33,0 \\
\hline & USF & 530 & 67,0 \\
\hline \multirow[t]{2}{*}{ Frequência de formação específica sobre MCP } & $\operatorname{Sim}$ & 293 & 34,9 \\
\hline & Não & 547 & 65,1 \\
\hline \multirow[t]{2}{*}{ Frequência de formação específica sobre consulta em MCP } & $\operatorname{Sim}$ & 264 & 31,5 \\
\hline & Não & 575 & 68,5 \\
\hline \multirow[t]{2}{*}{ Julga praticar MCP } & Sim & 673 & 79,9 \\
\hline & Não & 169 & 20,1 \\
\hline
\end{tabular}

Tabela 3 - Caracterização das dimensões encontradas após análise fatorial

\begin{tabular}{lccccc}
\hline \multicolumn{1}{c}{ Fator/ Dimensão } & Valor próprio & $\begin{array}{c}\text { Variância } \\
\text { explicada }\end{array}$ & Perguntas & $\begin{array}{c}\text { Alfa de } \\
\text { Cronbach }\end{array}$ & $\begin{array}{c}\text { M } \pm \text { dp da } \\
\text { dimensão }\end{array}$ \\
\hline 1: Anamnese & 7,002 & $31,8 \%$ & $2,3,4,5,6,12,13$ & 0,819 & $2,18 \pm 0,55$ \\
2: Investir na relação & 2,133 & $9,7 \%$ & $1,7,8,18,19,20,21,22$ & 0,783 & $1,51 \pm 0,39$ \\
3: Procurar entendimento & 1,482 & $6,7 \%$ & $14,15,16,17$ & 0,844 & $1,54 \pm 0,52$ \\
4: Pessoa como um todo & 1,242 & $5,6 \%$ & $9,10,11$ & 0,803 & $2,13 \pm 0,67$ \\
\hline
\end{tabular}

$\mathrm{M} \pm \mathrm{dp}$ : média \pm desvio padrão

Esta análise permitiu garantir as quatro dimensões definidas pela autora, com $53,9 \%$ da variância explicada. O teste KMO obteve o valor de 0,901 e o teste de esfericidade de Bartlett teve uma significância associada inferior a 0,001.

A Tabela 3 apresenta, para cada dimensão encontrada, o valor próprio associado, a percentagem de variância explicada e os itens que, efetivamente, estão incluídas nessa dimensão. Na última coluna desta tabela estão também apresentados os valores-sumário (média e desvio padrão) de cada dimensão.

Uma vez encontrados estes valores-sumário das quatro dimensões, foi possível determinar as correlações item-total (coluna 4 da Tabela 1), todas elas significativas ( $p$ $>0,01$ ). Nesta tabela, a seguir a estas correlações estão representadas entre parêntesis retos as dimensões a que cada item pode ser associado. Olhando para esta tabela e para as correlações item-total, verificamos que a dimensão 1 (anamnese) varia de 0,655 a 0,726, a dimensão 2 (investir na relação) varia de 0,457 a 0,755, a dimensão 3 (procurar entendimento) varia de 0,766 a 0,870, e a dimensão 4 (pessoa como um todo) varia de 0,830 a 0,870 .

Seguidamente, testaram-se as duas primeiras hipóteses relativas à validade de constructo. Pela análise da Tabela 4 podemos afirmar que as mulheres médicas estão mais sensibilizadas do que os seus colegas no que respei- ta à procura de entendimento com o doente. Em relação à idade é também evidente que são os médicos mais novos os mais sensíveis à abordagem da doença através da anamnese e, de novo, a procura de entendimento com o doente $\left(\mathrm{H}_{1}\right)$.

Do mesmo modo são os especialistas que mais cuidado têm com a anamnese e com o encarar o doente como um todo. Por outro lado, o local em que os cuidados são prestados é também um determinante para uma melhor adesão ao MCP. De facto, os profissionais que trabalham numa USF são os que procuram um melhor entendimento com a pessoa $\left(\mathrm{H}_{2}\right)$.

Esta mesma tabela permite-nos concluir os testes de validade de critério. Aqui a formação específica sobre MCP e sobre consulta em MCT demonstraram ter um impacto positivo em todas as dimensões na abordagem que os médicos seguem com vista a uma completa implementação do $\operatorname{MCP}\left(\mathrm{H}_{3}, \mathrm{H}_{4}\right)$, sendo isso reconhecido pelos próprios profissionais $\left(\mathrm{H}_{5}\right)$.

\section{DISCUSSÃO}

Neste estudo utilizou-se uma metodologia mista, incluindo metodologia qualitativa no grupo focal e metodologia quantitativa na análise de dados. ${ }^{12,15}$ Foi feita a clara opção de estudo em MGF por ser esta a especialidade que 
Tabela 4 - Sensibilidade do MCP a características socioeconómica e laborais

\begin{tabular}{|c|c|c|c|c|c|c|c|c|c|}
\hline \multirow{2}{*}{ Variável } & \multirow{2}{*}{ Valor } & \multicolumn{2}{|c|}{ Dimensão 1} & \multicolumn{2}{|c|}{ Dimensão 2} & \multicolumn{2}{|c|}{ Dimensão 3} & \multicolumn{2}{|c|}{ Dimensão 4} \\
\hline & & $M \pm d p$ & Sig & $M \pm d p$ & Sig & $M \pm d p$ & Sig & $M \pm d p$ & Sig \\
\hline Sexo & $\begin{array}{l}\text { Masculino } \\
\text { Feminino }\end{array}$ & $\begin{array}{l}2,13 \pm 0,58 \\
2,20 \pm 0,55\end{array}$ & 0,093 & $\begin{array}{l}1,53 \pm 0,42 \\
1,49 \pm 0,36\end{array}$ & 0,214 & $\begin{array}{l}1,61 \pm 0,58 \\
1,50 \pm 0,50\end{array}$ & 0,005 & $\begin{array}{l}2,18 \pm 0,71 \\
2,10 \pm 0,64\end{array}$ & 0,101 \\
\hline Idade & $\begin{array}{l}\leq 35 \text { anos } \\
] 35-55] \text { anos } \\
>55 \text { anos }\end{array}$ & $\begin{array}{l}2,07 \pm 0,58 \\
2,20 \pm 0,50 \\
2,27 \pm 0,60\end{array}$ & 0,002 & $\begin{array}{l}1,49 \pm 0,37 \\
1,51 \pm 0,37 \\
1,54 \pm 0,47\end{array}$ & 0,477 & $\begin{array}{l}1,52 \pm 0,50 \\
1,64 \pm 0,58 \\
1,63 \pm 0.62\end{array}$ & 0,031 & $\begin{array}{l}2,06 \pm 0,67 \\
2,11 \pm 0,62 \\
2,13 \pm 0,74\end{array}$ & 0,564 \\
\hline Local & $\begin{array}{l}\text { UCSP } \\
\text { USF }\end{array}$ & $\begin{array}{l}2,17 \pm 0,59 \\
2,20 \pm 0,54\end{array}$ & 0,431 & $\begin{array}{l}1,55 \pm 0,41 \\
1,49 \pm 0,37\end{array}$ & 0,039 & $\begin{array}{l}1,59 \pm 0,54 \\
1,52 \pm 0,52\end{array}$ & 0,104 & $\begin{array}{l}2,15 \pm 0,72 \\
2,13 \pm 0,64\end{array}$ & \\
\hline Especialização & $\begin{array}{l}\text { Especialista } \\
\text { Interno }\end{array}$ & $\begin{array}{l}2,13 \pm 0,55 \\
2,31 \pm 0,55\end{array}$ & $<0,001$ & $\begin{array}{l}1,50 \pm 0,39 \\
1,52 \pm 0,38\end{array}$ & 0,499 & $\begin{array}{l}1,56 \pm 0,54 \\
1,49 \pm 0,50\end{array}$ & 0,064 & $\begin{array}{l}2,08 \pm 0,67 \\
2,24 \pm 0,67\end{array}$ & 0,003 \\
\hline Formação em MCP & $\begin{array}{l}\text { Sim } \\
\text { Não }\end{array}$ & $\begin{array}{l}2,00 \pm 0,56 \\
2,26 \pm 0,53\end{array}$ & $<0,001$ & $\begin{array}{l}1,45 \pm 0,38 \\
1,54 \pm 0,38\end{array}$ & 0,001 & $\begin{array}{l}1,46 \pm 0,48 \\
1,59 \pm 0,55\end{array}$ & $<0,001$ & $\begin{array}{l}1,96 \pm 0,67 \\
2,21 \pm 0,65\end{array}$ & $<0,001$ \\
\hline Formação em consulta & $\begin{array}{l}\text { Sim } \\
\text { Não }\end{array}$ & $\begin{array}{l}2,00 \pm 0,57 \\
2,25 \pm 0,53\end{array}$ & $<0,001$ & $\begin{array}{l}1,45 \pm 0,38 \\
1,53 \pm 0,38\end{array}$ & 0,010 & $\begin{array}{l}1,45 \pm 0,47 \\
1,59 \pm 0,55\end{array}$ & $<0,001$ & $\begin{array}{l}1,98 \pm 0,68 \\
2,19 \pm 0,65\end{array}$ & $<0,001$ \\
\hline Julga praticar MCP & $\begin{array}{l}\text { Sim } \\
\text { Não }\end{array}$ & $\begin{array}{l}2,09 \pm 0,53 \\
2,51 \pm 0,53\end{array}$ & $<0,001$ & $\begin{array}{l}1,46 \pm 0,37 \\
1,68 \pm 0,41\end{array}$ & $<0,001$ & $\begin{array}{l}1,49 \pm 0,49 \\
1,76 \pm 0,61\end{array}$ & $<0,001$ & $\begin{array}{l}2,05 \pm 0,66 \\
2,43 \pm 0,63\end{array}$ & $<0,001$ \\
\hline
\end{tabular}

M: média; dp: desvio padrão; Sig: significância

nos seus fundamentos e mesmo na sua definição ${ }^{16}$ tem em atenção este método, sendo de realçar a atitude de colaboração de todos os entrevistados.

A consistência interna foi verificada e a fiabilidade pelo teste-reteste e pela análise das correlações item-total, permitiu determinar que os resultados obtidos são estáveis no tempo numa amostra alargada. A validação, efetuada em estudo populacional, permitiu verificar que há diferenças significativas em função de ter havido ou não formação específica quer em MCP, em consulta em MCP e por julgar realizar consulta em MCP.

É de salientar, no entanto, que a maioria desta amostra refere não ter tido frequência de qualquer formação específica na área, mas que 673 (79,9\%) têm a auto perceção de realizar consulta em $\mathrm{MCP}$.

Este é o primeiro instrumento para autoavaliação da realização de MCP. Existem outros instrumentos nesta área da MCP como a Medida de Comunicação Centrada na Pessoa, baseado em entrevistas áudio-gravadas, a Perceção da Pessoa Quanto ao Atendimento Centrado na Pessoa (PPACP) de nove itens, que mede os três primeiros componentes do MCP e que consiste num questionário dirigido aos doentes, ${ }^{12}$ e o Questionário da Medicina Centrada no Doente em Portugal (MCP-PT) ${ }^{11}$ que é destinado aos doentes e abrange os seis componentes de MCP, tal como em versão anterior descritos por Moira Stewart.

O presente questionário não se restringe à prática da consulta, permitindo avaliar melhor aspetos da relação médico-doente que exigem tempo para se estabelecerem e que não podem ser medidos a cada encontro. Sendo discutível que a satisfação da pessoa que consulta constitua, por si só, um bom indicador de qualidade dos cuidados de saúde, ${ }^{17}$ este questionário dirigido a médicos pode permitir mudar o atual paradigma essencialmente biomédico. ${ }^{18-20}$
Este instrumento assume a autoavaliação médica como parâmetro de mensuração do exercício da MCP e, se aplicado conjuntamente com outras ferramentas, permitirá certamente verificar a relação entre a abordagem centrada na pessoa e indicadores em saúde. É assim um indicador das necessidades de desenvolvimento profissional contínuo.

\section{CONCLUSÃO}

Foi possível desenvolver um instrumento que assume a autoavaliação médica como parâmetro de mensuração do exercício da MCP e simultaneamente é indicador das necessidades de desenvolvimento profissional contínuo.

\section{AGRADECIMENTOS}

Aos membros do grupo focal, ao responsável informático na Secção Regional do Centro da Ordem dos Médicos, às Coordenações de Internato em Medicina Geral e Familiar das Regiões Norte e Centro e a todos os que responderam ao trabalho proposto.

\section{PROTECÇÃO DE PESSOAS E ANIMAIS}

Os autores declaram que os procedimentos seguidos estavam de acordo com os regulamentos estabelecidos pelos responsáveis da Comissão de Investigação Clínica e Ética e de acordo com a Declaração de Helsínquia da Associação Médica Mundial.

\section{CONFIDENCIALIDADE DOS DADOS}

Os autores declaram ter seguido os protocolos do seu centro de trabalho acerca da publicação de dados.

\section{CONFLITOS DE INTERESSE}

Os autores declaram não ter conflitos de interesses relacionados com o presente trabalho. 


\section{FONTES DE FINANCIAMENTO}

Este trabalho não recebeu qualquer tipo de suporte

\section{REFERÊNCIAS}

1. Sacristán JA. Patient-centered medicine and patient-oriented research: improving health outcomes for individual patients. BMC Med Inform Decis Mak. 2013;13:6.

2. Draeger RW, Stern PJ. Patient-centered care in medicine and surgery. Hand Clin. 2014;30:353-9.

3. Stewart M, Brown J, Weston W, McWhinney I, McWilliam C, Freeman T. Patient-centered medicine: transforming the clinical method. 3rd ed. London: Radcliffe Publishing Ltd; 2014. p.313-30.

4. Pires P. Medicina centrada no paciente: melhor qualidade com menores custos. Rev Port Med Geral Fam. 2011;27:482-6.

5. Robinson J, Callister L, Berry J, Dearing K. Patient-centered care and adherence: definitions and applications to improve outcomes. J Am Acad Nurse Pract. 2008;20:600-7.

6. Rathert C, Wyrwich M, Boren S. Patient-centered care and outcomes: a systematic review of the literature. Med Care Res Rev. 2013;70:351-79.

7. Bertakis K, Azari R. Patient-centered care is associated with decreased health care utilization. J Am Board Fam Med. 2011;24:229-39.

8. Stewart M, Brown J, Donner A, McWhinney I, Oates J, Weston W, et al. The impact of patient-centered care on outcomes. J Fam Pract. 2000;49:796-804.

9. Gluyas H. Patient-centered care: improving healthcare outcomes. Nurs Stand. 2015;30:50-9.

10. Mendes-Nunes J. Comunicação em contexto clínico. Bayer Health Care. 2010:59-67.

11. Reis A, Santiago L, Botas P. Medicina centrada no paciente e financeiro de nenhuma entidade no domínio público ou privado.

capacitação do consulente em Medicina Geral e Familiar. Revista ADSO. 2015:19-32.

12. Stewart M, Brown J, Weston W, McWhinney I, McWilliam C, Freeman T. Patient-centered medicine: transforming the clinical method. 3rd ed. London: Radcliffe Publishing Ltd; 2014. p. 331-7.

13. Terwee C, Bot S, de Boer M, van der Windt D, Knol D, Dekker J, et al. Quality criteria were proposed for measurement properties of health status questionnaires, J Clin Epidemiol. 2007;60:34-42.

14. Lisboa J, Augusto M, Ferreira P. Estatística aplicada à gestão. Porto: Vida Económica; 2012.

15. Koo T, Li M. A guideline of selecting and reporting intraclass correlation coefficients for reliability research. J Chiropr Med. 2016;15:155-63.

16. Silva D. Helping measure person-centered care: a review of evidence about commonly used approaches and tools used to help measure person-centered care. London: The Health Foundation; 2014.

17. Wonca Europe. The European Definition of General Practice / Family Medicine. [consultado 2018 mar 20]. Disponível em: http:// www.woncaeurope.org/sites/default/files/documents/Definition $\% 20$ EURACTshortversion.pdf.

18. Heidenreich P. Time for a thorough evaluation of patient-centered care. Circ Cardiovasc Qual Outcomes. 2013;6:2-4.

19. Bardes C. Defining "Patient-Centered Medicine". N Engl J Med. 2012;366:782-3.

20. Epstein R, Street $R$ Jr. The values and value of patient-centered care. Ann Fam Med. 2011;9:100-3. 